\title{
Compte rendu d'Indigenising Anthropology with Guattari and Deleuze de Barbara Glowczewski
}

\section{Céline Travési}

\section{OpenEdition}

1 Journals

Édition électronique

URL : https://journals.openedition.org/jso/11651

DOI : 10.4000/jso.11651

ISSN : $1760-7256$

Éditeur

Société des océanistes

\section{Édition imprimée}

Date de publication : 8 juin 2020

Pagination : 109-111

ISBN : 9782854301380

ISSN : 0300-953x

\section{Référence électronique}

Céline Travési, « Compte rendu d'Indigenising Anthropology with Guattari and Deleuze de Barbara Glowczewski », Journal de la Société des Océanistes [En ligne], 150 | 2020, mis en ligne le 08 juin 2020, consulté le 06 janvier 2022. URL : http://journals.openedition.org/jso/11651 ; DOI : https://doi.org/ 10.4000/jso. 11651

Ce document a été généré automatiquement le 6 janvier 2022.

\section{cc) (†) $\ominus$}

Journal de la société des océanistes est mis à disposition selon les termes de la Licence Creative Commons Attribution - Pas d'Utilisation Commerciale - Pas de Modification 4.0 International. 


\title{
Compte rendu d'Indigenising Anthropology with Guattari and Deleuze de Barbara Glowczewski
}

\author{
Céline Travési
}

\section{RÉFÉRENCE}

GLOWCZEWSKI Barbara, 2020. Indigenising Anthropology with Guattari and Deleuze,

Edinburgh, Edinburgh University Press, 446 p., bibliogr., index, ill., fig.

1 Le Pacifique est sans doute aujourd'hui l'un des principaux lieux « où se renégocient les conditions sociales, administratives et intellectuelles de la recherche " (Trépied, 2011 : 159). Que ce soit en Nouvelle-Calédonie (Trépied, 2011), en Australie (de Largy Healy, 2011), en Nouvelle-Zélande (Smith, 1999; Gagné, 2008), ou ailleurs en Océanie, les chercheurs sont aujourd'hui confrontés à une nécessité qui est celle de la décolonisation des sciences sociales. Dans ce contexte, Le dernier ouvrage de Barbara Glowczewski, Indigenising anthropology with Guattari and Deleuze, mérite toute notre attention.

Le livre propose une compilation, en langue anglaise, d'une partie des écrits de l'auteure. Il réunit, articule et relie de manière éclairante et particulièrement inspirante un certain nombre de ses travaux (articles, conférences, extraits d'ouvrages), et permet de redécouvrir sous un angle nouveau et englobant le travail de cette grande anthropologue australianiste française, spécialiste des Aborigènes, ainsi que celui de Félix Guattari, avec lequel elle engagea très tôt un dialogue - sans doute l'un des plus stimulants du point de vue des sciences sociales contemporaines. Ce livre n'est pas une simple collection de morceaux choisis. C'est un manifeste. Il se présente à la fois comme le bilan d'une œuvre - ou plutôt celui d'œuvres différentes (celle de l'auteure, celles de Gilles Deleuze et de Félix Guattari) - et comme une profession de foi, dans laquelle l'anthropologue nous invite à nous emparer du projet intellectuel et 
humaniste, qui est à la fois celui de Félix Guattari et des Aborigènes. En ce sens, l'ouvrage proposé par Barbara Glowczewski représente une contribution importante et nécessaire, pour l'anthropologie mais aussi, plus généralement, pour toutes les sciences sociales et le projet qui les fonde.

L'auteur se livre sur son parcours, sur son cheminement professionnel et intellectuel mais aussi personnel. Pour autant, le livre n'est pas une autobiographie. C'est le parcours d'une pensée qui est retracé ici, ou plutôt celui de plusieurs pensées qui se croisent, s'interpellent, se répondent. L'ouvrage introduit notamment de nouvelles perspectives et apporte un éclairage nouveau sur les travaux de Gilles Deleuze et de Félix Guattari, bien connus dans le monde anglo-saxon. La mise en perspective des concepts développés par ces auteurs avec les cosmopolitiques aborigènes représente un aspect très présent dans les travaux de Barbara Glowczewski qui n'avaient été que partiellement traduits jusqu'ici. Le fait de les rassembler dans une même publication accessible, voire dédiée, aux lecteurs anglophones, représente une manière de continuer à enrichir la compréhension et l'apport de la pensée de ces philosophes sur la scène internationale.

Le premier chapitre, "Becoming Land", introduit et met en perspective les différents chapitres en les reliant au parcours de l'auteure, à ses rencontres, à la pensée de Guattari et aux cosmopolitiques aborigènes. Il revient sur la rencontre et les échanges entre l'anthropologue et le philosophe et sur leurs influences réciproques. Alors que Guattari puise dans le travail et les données de Barbara Glowczewski de quoi illustrer, enrichir et faire évoluer ses propres travaux, celle-ci réalise combien ce qu'elle découvre chez les Aborigènes et ce qu'elle écrit fait écho aux concepts de rhizome (Guattari, 1989), de différence et de répétition (Deleuze, 1968), de transversalité et de dissensus (Guattari, 1972). La composition du livre, divisé en cinq grandes sections, s'articule en partie autour de ces concepts qui traversent aussi l'ensemble des chapitres. L'influence, ou plutôt la rencontre avec la pensée de Guattari, en particulier, est partout. Elle traverse l'ouvrage et les travaux de l'auteure et l'accompagne sur le terrain, jusqu'au Brésil, où le célèbre philosophe et psychanalyste a aussi marqué toute une génération de chercheurs et d'artistes.

5 L'ouvrage est donc également un hommage - à Félix Guattari, mais aussi aux Aborigènes, et plus largement, aux peuples autochtones. Chacun d'entre eux représente des acteurs et des voix qu'il s'agit de faire dialoguer, comme lorsque le méta-modèle cartographique de Guattari ou son approche thérapeutique sont confrontés aux concepts d'un homme warlpiri (chapitre 13) ou à ceux d'un guérisseur yalarrnga (chapitre 14). Barbara Glowczewski montre alors comment ces modes de pensée, déployés de manière similaire, se répondent pour "approcher l'inconscient collectif comme une forme d'intersubjectivation et de force active permettant de faire bouger le monde » (p. 64, ma traduction). Pour l'anthropologue, l'objectif consiste à restituer les voix du «multiverse » qu'elle explore, celles des autochtones, celles de Deleuze et Guattari, et celles d'autres collègues anthropologues, penseurs, activistes; des voix qu'elle laisse aussi s'exprimer librement sous la forme de longues citations, d'extraits d'ouvrages (dans le cas de Guattari), ou dans le prélude qui est la transcription de la narration d'un rêve de Nakakut Barbara Gibson Nakamarra, une femme aborigène warlpiri.

6 Avec cet ouvrage, Barbara Glowczewski nous propose une anthropologie « qui échappe à la représentation » (p. 61). En mêlant les genres, en donnant à entendre, à voir et à 
sentir, dans l'imbrication qui les définit, les voix, les pensées et les expériences multiples d'acteurs qui ont ou auraient tant à se dire, elle nous livre sa proposition pour une anthropologie «indisciplinée». Barbara Glowczewski est une adepte des projets expérimentaux. Elle a ainsi initié ou collaboré à de nombreux projets innovants, le plus souvent interactifs, expérimentant toujours de nouveaux types de supports, de formats ou de contenus ( $c f$. chapitre 9 et 13), avec un intérêt particulier pour les films expérimentaux (encore un intérêt commun avec Guattari). C'est aussi cela, « indigéniser l'anthropologie ». Ce goût pour l'expérimentation se retrouve aussi dans la forme originale donnée à ce livre, dont la composition réticulaire faite d'« hyperliens ", et de lignes ou de voix qui s'entrecroisent - que l'on retrouve, quoique sous une forme différente, dans Rêves en colère (Glowczewski, 2004) - restitue bien les pensées articulées en réseau de Guattari et des Aborigènes. L'auteure explique comment, à un moment de sa carrière, certains collègues l'accusèrent de "s'indigéniser » ("going native »), quand elle avoue avoir été plus inspirée par les Aborigènes eux-mêmes (ce qui est aussi le cas de Guattari lorsqu'il s'inspire de ce que Barbara Glowczewski écrit au sujet des Aborigènes). Or il s'agit bien là d'une décolonisation de la pensée dont les sciences sociales gagneraient aujourd'hui largement à s'inspirer.

7 L'ouvrage se présente en effet surtout comme un projet intellectuel et humaniste, résonnant comme un appel à nous tenir, nous aussi, « debout avec la Terre » (chapitre 12) et à " résister au désastre » (chapitre 11). Le livre aurait tout aussi bien pu avoir pour sous-titre : «Pour une nouvelle cosmopolitique des sciences sociales» - au sens entendu par Stengers (2005). Barbara Glowczewski appelle, dans la continuité du projet écosophique de Félix Guattari $(1992,2013)$, à une « refondation des pratiques sociales » dans laquelle les sciences sociales ont un rôle à jouer. L'un des projets de la recherche pourrait ainsi consister à « valoriser et disséminer l'information au sujet des solutions alternatives et expérimentales qui peuvent être éprouvées à travers le monde » (p. 43, ma traduction) et ainsi, refonder un espoir partagé face aux enjeux contemporains qui se posent quant au destin de la planète. On pourrait regretter qu'il ne soit pas fait davantage état de la dimension sensible de l'expérience sur laquelle s'appuie aussi l'indigénisation de l'anthropologie et que l'auteure évoque à la fin du prélude (p.4). Mais cela ne diminue en rien l'intérêt de l'ouvrage, dont la portée, à la fois universelle et singulière, doit, comme les travaux de Félix Guattari ou la pensée des Aborigènes, continuer à nous inspirer.

\section{BIBLIOGRAPHIE}

DELEUZE Gilles, 1968. Différence et répétition, Paris, PUF.

DELEUZE Gilles et Félix GUATTARI, 1972. Capitalisme et schizophrénie : l'anti-œdipe, Paris, éditions de Minuit.

GAGNÉ Natasha, 2008. Le savoir comme enjeu de pouvoir. L'ethnologue critiquée par les autochtones, in A. Bensa et D. Fassin (éds), Les politiques de l'enquête. Épreuves ethnographiques, Paris, La Découverte, pp. 277-298. 
GLOWCZEWSKI Barbara, 2004. Rêves en colère : alliances aborigènes dans le Nord-Ouest australien, Paris, Plon/Terre Humaine.

GUATTARI Félix, 1972. Psychanalyse et transversalité : essais d'analyse institutionnelle, Paris, Maspero. GUATTARI Félix, 1989. Cartographies schizoanalytiques, Paris, Galilée.

GUATTARI Félix, 1992 (oct.). Pour une refondation des pratiques sociales, Le Monde diplomatique, pp. 26-27.

GUATTARI Félix, 2013. Qu'est-ce que l'écosophie ? textes présentés par Stéphane Nadaud, Paris, Nouvelles éditions Lignes, IMEC, coll. Archives de la pensée critique.

DE LARGY HEALY Jessica, 2011. Pour une anthropologie de la restitution. Archives culturelles et transmission des savoirs en Australie, Cahiers d'ethnomusicologie 24, pp. 45-65.

SMITH Linda Tuhiwai, 1999. Decolonizing Methodologies: Research and Indigenous Peoples, Londres et Dunedin, Zed Books et University of Otago Press.

STENGERS Isabelle, 2005. The cosmopolitical proposal, in B. Latour et P. Weibel (eds) Making Things Public, Cambridge, The MIT Press, pp. 994-1003.

TRÉPIED Benoît, 2011. Recherche et décolonisation en Nouvelle-Calédonie contemporaine : lectures croisées, Revue d'Histoire des Sciences Humaines 24 (1), pp. 159-187.

\section{AUTEUR}

\section{CÉLINE TRAVÉSI}

Chercheuse associée au CREDO, Aix-Marseille Université, CNRS, EHESS, CREDO UMR 7308, 13003, Marseille, celine.travesi@univ-amu.fr 